\title{
Mixture state of individual Asian dust particles at a coastal site of Qingdao, China
}

\author{
Daizhou Zhang ${ }^{\mathrm{a}, *}$, Jiaye Zang ${ }^{\mathrm{b}}$, Guangyu Shic, Yasunobu Iwasaka ${ }^{\mathrm{d}}$, \\ Atsushi Matsuki, Dmitri Trochkine \\ ${ }^{a}$ Faculty of Environmental and Symbiotic Sciences, Prefectural University of Kumamoto, Tsukide 3-1-100, Kumamoto 862-8502, Japan \\ ${ }^{\mathrm{b}}$ First Institute of Oceanography, State Oceanic Administration, Qingdao 266061, China \\ ${ }^{\mathrm{c}}$ Institute of Atmospheric Physics, Chinese Academy of Science, Beijing 100029, China \\ ${ }^{\mathrm{d}}$ Graduate School of Environmental Studies, Nagoya University, Nagoya 464-8601, Japan \\ ${ }^{\text {e }}$ Solar Terrestrial Environment Laboratory, Nagoya University, Nagoya 464-8601, Japan
}

Received 9 April 2003; received in revised form 3 June 2003; accepted 6 June 2003

\begin{abstract}
In order to investigate the characteristics of Asian dust particles before they leave the continent, particles were collected at a coastal site of Qingdao $\left(120^{\circ} 27^{\prime} \mathrm{E}, 36^{\circ} 06^{\prime} \mathrm{N}\right)$ in northeastern China during three dust storm events in spring 2001. The elemental composition and size of individual dust particles, and their mixture state with sea salt, sulfate and nitrate were analyzed using electron microscopes and an energy dispersive spectrometer. It was found that far less than $10 \%$ of dust particles were internally mixed with sea salt. Although a number of dust particles contained $\mathrm{Na}, \mathrm{S}$, and $\mathrm{Cl}$, the elements in most of the particles were more likely from crustal origins. The combination of reagent tests and elemental analysis revealed that $3.3-12.2 \%$ of dust particles contained sulfate and $6.5-10.0 \%$ contained nitrate on their surface. Number-size distributions of dust particles estimated from their electron microscope images had a distribution mode of around $3 \mu \mathrm{m}$ diameter with the range of $1.0-8.0 \mu \mathrm{m}$. Out of this range, dust particles were rarely detected.
\end{abstract}

(C) 2003 Elsevier Ltd. All rights reserved.

Keywords: Asian dust; Size distribution; Sulfate; Nitrate; Coastal area; Electron microscopy

\section{Introduction}

Due to westerly winds, dust particles from the arid and semi-arid areas of Asian continent are frequently transported out of the continent, reach the northern central Pacific, and sometimes even arrive at North America in spring (Duce et al., 1980; Uematsu et al., 1983; Iwasaka et al., 1988; Husar et al., 2001). As they disperse in the atmosphere, processes such as adsorbing gaseous species, surface reactions and coagulation with other particulate matter can change the composition and

\footnotetext{
*Corresponding author. Tel.: +81-96-383-2929; fax: +8196-384-6765.

E-mail address: zdz@pu-kumamoto.ac.jp (D. Zhang).
}

morphology of the particles. Additionally, the particles influence the atmospheric mass cycles associated with trace gases such as $\mathrm{SO}_{2}, \mathrm{NO}_{x}$ and $\mathrm{HCl}$ through these processes (Zhang et al., 1994; Dentener et al., 1996; Song and Carmichael, 2001; Zhang and Iwasaka, 2001). By reflecting and absorbing solar radiation and modifying cloud properties, dust particles may be important for climate change (Sokolik and Toon, 1996). Thus understanding the physical and chemical characteristics of Asian dust particles during their dispersion is important for determining their effects on the geochemical mass cycle and radiative forcing in this region.

Previous studies by bulk sample analysis at Japan revealed that particulate sulfate and nitrate concentrations increased by 3-10 times during dust episodes 
(Nishikawa et al., 1991; Kanamori et al., 1991). Okada et al. (1990) and Yamato and Tanaka (1994) confirmed the existence of sulfate and nitrate in dust particles and particles mixed with mineral components and sea salt around Japan by single-particle analysis. However, similar characteristics were rarely detected at and near dust source areas (Okada and Kai, 1995; Zhang and Iwasaka, 1999). This suggests that dust particles usually experience significant modification as they disperse from the source areas to Japan.

Before arriving at Japan, dust particles pass over land areas of northeastern and eastern China and the ocean areas between China and Japan (the Yellow Sea, the East China Sea and/or the Sea of Japan). Measurements in China during non-dust periods revealed that mineral particles could promote the heterogeneous formation of sulfate and nitrate by providing reaction sites in a polluted urban atmosphere (Zhang et al., 1995, 2000; Zhang, 1996). Anthropogenic emissions contribute significant pollutants in East Asia (Kato and Akimoto, 1992; Akimoto and Narita, 1994; Guttikunda et al., 2003). So an urgent concern is whether dust particles can mix with and carry such pollutants when they disperse in the continental atmosphere. To answer this question, the information of dust particles at the transition zone from the continent to the ocean, e.g. the coastal areas of China, is essential.

In 2001, we observed dust particles at Qingdao, a coastal city in northeastern China, where Asian dust storms frequently pass to leave the continent in spring. In this paper, results of dust particles from three Asian dust events are reported. The main purpose of this study is to provide exact physical and chemical characteristics of Asian dust particles just before they leave the continent.

In this study, particles were analyzed individually using electron microscopes and an energy dispersive X-ray (EDX) spectrometer. Morphologies, geometric size and elemental composition of individual particles were obtained. With reagents on sampling film, the presence of sulfate and nitrate in individual particles was also identified. Compared to bulk sample analysis, by which only the average information of total collected particulate matter is available, single-particle analysis allows the exact characteristics of each particle to be obtained and such data are more useful for identifying particle changes.

\section{Particle collection and analysis}

Dust particles were collected during three dust storm events at Qingdao (named as Q-1, Q-2, and Q-3) in spring 2001. The sampling time and weather conditions are listed in Table 1. Fig. 1 shows the position of Qingdao and the isentropic backward trajectories of the observed dust events. The sampling site is on the roof of the main building of the First Institute of Oceanography of the State Oceanic Administration of China $\left(120^{\circ} 27^{\prime} \mathrm{E}\right.$, $\left.36^{\circ} 06^{\prime} \mathrm{N}\right)$, which is about $35 \mathrm{~m}$ above the ground and located at the seashore near the Yellow Sea. Particles were collected onto electron microscope Pt grids $(3 \mathrm{~mm}$ diameter) and formvar film-covered copper meshes (Maxtaform H7) using a single-stage cascade impactor. Before particle collection, the film on the meshes was coated with nitron and barium chloride reagents for the

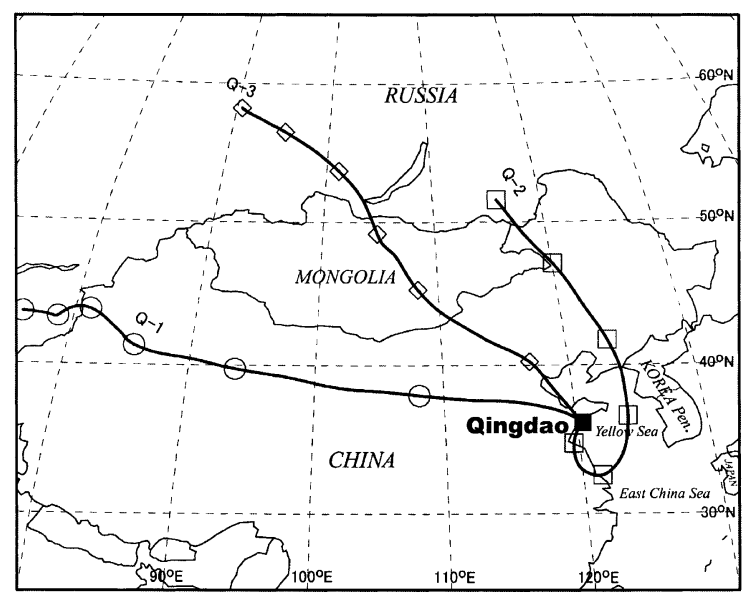

Fig. 1. Isentropic backward trajectories of the three dust events observed at Qingdao. The calculation was performed on-line by the HYSPLIT model of NOAA Air Resources Laboratory's web server. The trajectories start at the altitude of $1000 \mathrm{~m}$ above the sampling site and from the sampling time. Marks on the trajectories mark 12-h increments.

Table 1

Summary of sampling time and surface weather conditions at the sampling sites when particles were collected

\begin{tabular}{|c|c|c|c|c|c|c|}
\hline \multirow[t]{2}{*}{ Dust event } & \multicolumn{2}{|c|}{ Sampling time $\left(\mathrm{BST}^{\mathrm{a}}\right)$} & \multirow[t]{2}{*}{$\mathrm{RH}(\%)$} & \multirow[t]{2}{*}{ Visibility (km) } & \multicolumn{2}{|l|}{ Wind } \\
\hline & Date & Period & & & Direction & Speed $\left(\mathrm{m} \mathrm{s}^{-1}\right)$ \\
\hline Q-1 & 6 March 2001 & $11: 15-12: 30$ & 20 & $\sim 3$ & NW & $\sim 10$ \\
\hline Q-2 & 23 April 2001 & $15: 00-16: 00$ & 22 & $\sim 5$ & $\mathrm{~N}$ & $\sim 10$ \\
\hline Q-3 & 15 May 2001 & $12: 30-13: 30$ & 24 & $\sim 10$ & $\mathrm{~N}$ & $\sim 6$ \\
\hline
\end{tabular}

\footnotetext{
${ }^{\text {a }}$ Beijing Standard Time $(=\mathrm{GMT}+8 \mathrm{~h})$.
} 
detection of nitrate and sulfate in particles. Details of the reagent coating can be found in Qian et al. (1991), except that both reagents were pre-coated before particle collection. The jet diameter of the impactor was $1 \mathrm{~mm}$ and the flow rate of inlet air was $51 \mathrm{~min}^{-1}$. Assuming that the particle density is $2.3 \mathrm{~g} \mathrm{~cm}^{-3}$, the $50 \%$ cutoff diameter of the impactor is about $0.25 \mu \mathrm{m}$ and all particles larger than $0.6 \mu \mathrm{m}$ can be captured by this system. The collection time for each grid was $2 \mathrm{~min}$. After collection, each grid was kept in a plastic capsule, which, in turn, was sealed in plastic bags together with paper-packaged silica gel.

Particles on $\mathrm{Pt}$ grids were investigated and photographed using the scanning electron microscope (SEM; Hitachi S-3000N) of the Solar Terrestrial Environment Laboratory of Nagoya University for the observation of particle morphology and size. The pictures covered the central regions where particles were collected on the grids. After a picture was taken, the elemental composition of every particle in the picture was determined using the EDX spectrometer (Horiba EMAX-500) attached to the SEM. With this SEMEDX system, we were able to quantitatively detect the relative weight and atom ratios of elements $(Z>5$ except nitrogen) in a single particle down to $0.1 \mu \mathrm{m}$. The SEM was operated at $20 \mathrm{keV}$ accelerating voltage and $80 \mu \mathrm{A}$ filament current. The $\mathrm{X}$-ray spectrum of a particle was generated from a square covering the particle and was integrated for 50 live seconds with probe current of around $0.3 \mathrm{nA}$. The atom number fractions and weight fractions of different elements in individual particles were calculated using $Z A F$ ( $Z$ : element atomic number; $A$ : X-ray absorption; $F$ : X-ray fluorescence) matrix correction. To avoid underestimating the content of sodium $(\mathrm{Na})$, low-energy correction was not applied in the ratio calculation, which led to unreasonably large ratios of carbon and oxygen and overestimated ratios of $\mathrm{Na}$. In terms of elemental composition, dust particles are those mainly containing silicon $(\mathrm{Si})$ together with/without other mineral elements, such as aluminum (Al) and iron (Fe). Sea salt particles are those abundant in $\mathrm{Na}$ and chlorine $(\mathrm{Cl})$ and containing minor sulfur (S) and magnesium (Mg), but no $\mathrm{Si}, \mathrm{Al}$ or Fe (Eriksson, 1959; Posfai et al., 1996).

Before analyzing particles collected on meshes coated with reagents, the meshes were exposed to octanol vapor for $18 \mathrm{~h}$ at room temperature to promote the reactions of sulfate and/or nitrate with the reagent film. Particles on the films were viewed and photographed using the Hitachi H-9 transmission electron microscope (TEM) of the Hydrospheric-Atmospheric Research Center (HyARC) of Nagoya University. From the TEM images, particles containing sulfate on their surface can be identified by the appearance of Liesegang rings of barium sulfate, the product of the reaction of sulfate with barium chloride, and particles containing nitrate by the appearance of bundles of needle-like crystals of nitron nitrate, the product of the reaction of nitrate with nitron. Particles containing both sulfate and nitrate can be identified by the simultaneous appearance of bariumsulfate rings and nitron-nitrate bundles around the particles. According to the laboratory study of Qian et al. (1991), the detection limits of the reagent film for sulfate and nitrate contained in individual particles are $10^{-17}$ and $10^{-14} \mathrm{~g}$, respectively.

\section{Results and discussion}

The backward trajectories in Fig. 1 indicate that Q-1 was from northwestern China, Q-2 was from northeastern China, and Q-3 was from Mongolia and northern China. Although the trajectory of Q-2 indicates that the air masses passed marine areas before arriving at the sampling site, the collected dust particles during this event, as described in the following sections, were similar to those of the other two events and no apparent marine influences were detected, suggesting that the air masses were not considerably influenced by marine air, even though they might have had a short experience over the ocean or the trajectory was not the exact pathway of the air masses. In addition, mineral particles from local areas, if there were such particles in the samples, could be neglible because no significant sources emitting mineral particles such as road dust were expected near the sampling site. So to a large extent, we believe that the mineral particles collected during the three events can represent the dust particles arriving at Qingdao through long-range transport.

\subsection{Elemental composition and mixture with sea salt}

Fig. 2 illustrates the number fractions of dust particles containing different elements in the total detected dust particles. The particles mainly contained $\mathrm{Mg}, \mathrm{Al}, \mathrm{K}, \mathrm{Ca}$,

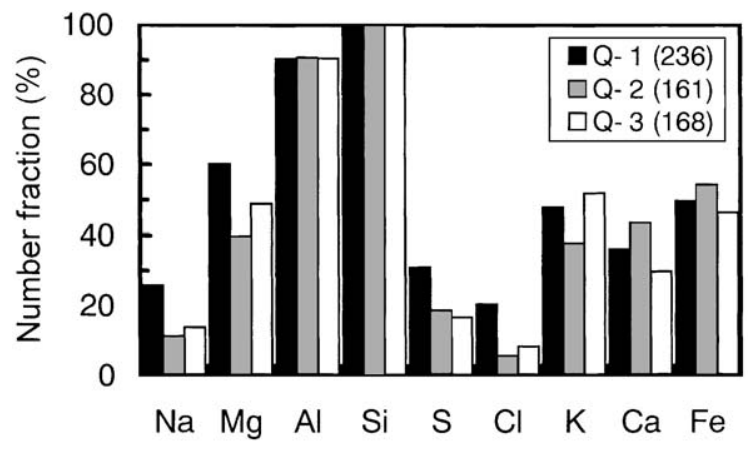

Fig. 2. Number fractions of dust particles containing different elements in total detected dust particles of the three dust storm events. The number of dust particles detected in each event are listed in parentheses. 
and $\mathrm{Fe}$ besides $\mathrm{Si}$, which is similar to the composition of Asian dust particles at source areas (Nishikawa et al., 2000). A number of dust particles contained $\mathrm{Na}$ and $\mathrm{Cl}$ besides the above mineral elements. The fractions of $\mathrm{Na}-$, S- and Cl-containing dust particles are $11.2-25.8 \%$, $16.7-30.9 \%$ and $5.6-20.3 \%$, respectively. These data are approximately at the same levels as those previously observed in Beijing, an inland city located in upwind of Qingdao, during dust storm events (Zhang and Iwasaka, 1999). If we separate dust particles into two groups, "Na\&Cl particles" and "non- $\mathrm{Na} \& \mathrm{Cl}$ particles", $\mathrm{Na \& Cl}$ particles are dust particles containing both $\mathrm{Na}$ and $\mathrm{Cl}$, and the remaining are non- $\mathrm{Na \& Cl}$ particles, the fractions of $\mathrm{Na \& Cl}$ particles comprise less than $20 \%$, average $9.9 \%$ (Fig. 3). Since the dust particles dispersed from inland areas to the coastal areas in the continental atmosphere and the wind at Qingdao when the particles were collected were from the land to the sea (Table 1 and Fig. 1), sea salt contributions of $\mathrm{Na}$ and $\mathrm{Cl}$ might be small and need to be identified in order to access the crustal contributions of these elements.

The weight ratios of $\mathrm{Na}: \mathrm{S}: \mathrm{Cl}$ in individual dust particles and the relative weight levels of $\mathrm{Na}, \mathrm{S}$ and $\mathrm{Cl}$ are shown in Fig. 4. For reference, the region of fresh sea salt particles is plotted in Fig. 4a. The ratios of Na:S:Cl in most particles are much different from sea salt. It is noted that $\mathrm{S}$ occupies much larger fractions of weight than $\mathrm{Na}$ and $\mathrm{Cl}$ in a number of particles although the ratios of $\mathrm{Na} / \mathrm{Cl}$ in some of them are close to that of sea salt. Ratios of Na:S:Cl in merely a few particles are close to that of sea salt. Two speculative reasons can account for these results. One is that $\mathrm{Na}$ and $\mathrm{Cl}$ in most particles are not from sea salt. Instead, they originated from dust sources, i.e. crustal origins. Another is that $\mathrm{Na}$ and $\mathrm{Cl}$ are originally from sea salt and, in that sense, $\mathrm{S}$ deposition has occurred on the particles and resulted in significant $\mathrm{Cl}$ loss. It has been confirmed that some dust particles originally contain $\mathrm{Na}$ and $\mathrm{S}$, and their compounds are almost completely water-soluble

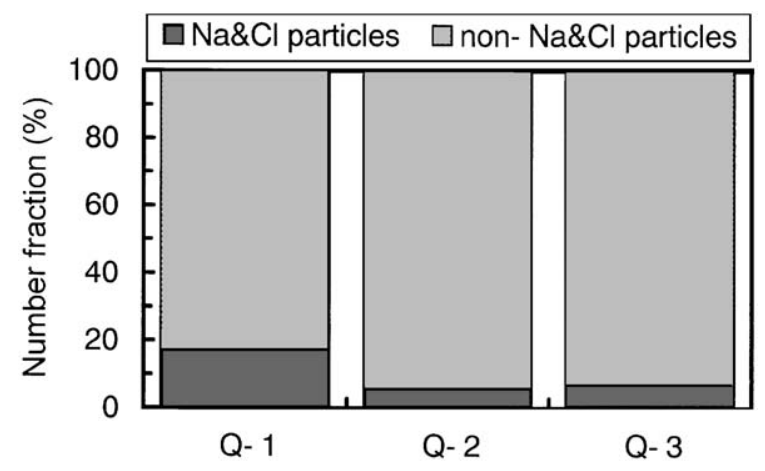

Fig. 3. Number fractions of $\mathrm{Na} \& \mathrm{Cl}$ dust particles and non$\mathrm{Na} \& \mathrm{Cl}$ dust particles in detected dust particles of the three dust storm events.

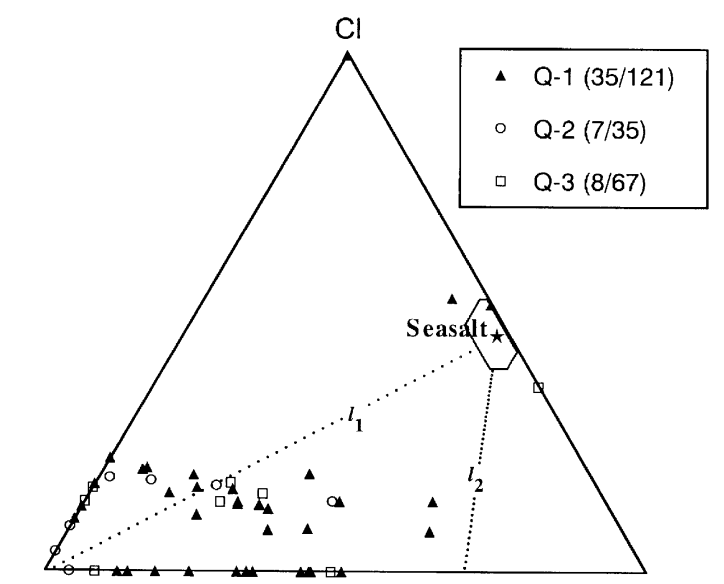

$\mathrm{s}$

(a)

$\mathrm{Na}$

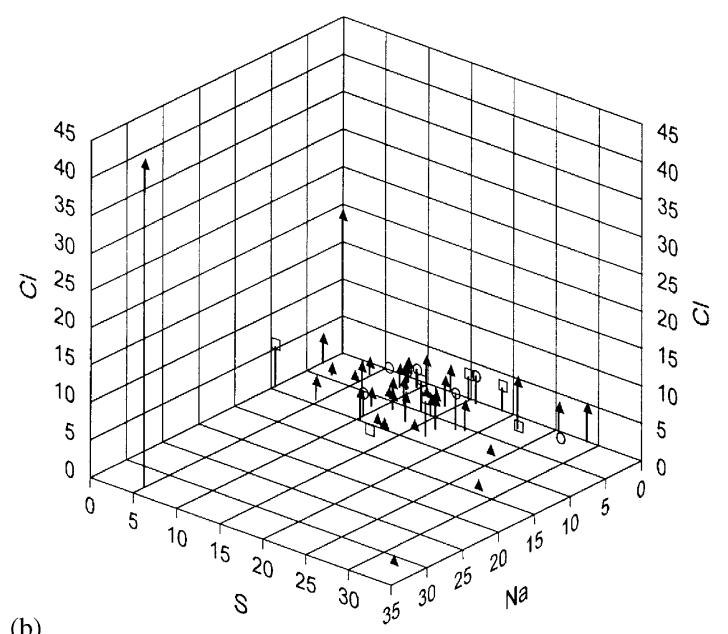

(b)

Fig. 4. (a) The relative weight ratios of $\mathrm{Na}: \mathrm{S}: \mathrm{Cl}$, and (b) the absolute levels of $\mathrm{Na}, \mathrm{S}$ and $\mathrm{Cl}$ relative weight ratios in individual dust particles which contained at least two $\mathrm{Na}, \mathrm{S}$ and $\mathrm{Cl}$. For reference, we measured fresh sea salt particles collected near the surface of the Sea of Japan when only natural sea salt particles were expected using the same analysis equipments and methods under the same conditions. In (a), the star shows the position of average Na:S:Cl of the sea salt particles and the hexagon circling the star shows the range of the detected sea salt particles. If a particle appears in the hexagon, the sea salt content in it is not modified. $l_{1}$ is the line along which $\mathrm{Na} / \mathrm{Cl}$ is constant as in fresh sea salt, and $l_{2}$ is the line along which $\mathrm{Na}: \mathrm{S}: \mathrm{Cl}$ is changed only by reactions of every $2 \mathrm{Cl}$ replaced by $1 \mathrm{~S}$. Figures in parentheses are (dust particles appearing in the triangle)/(quantitatively detected dust particles) for each event.

(Nishikawa et al., 2000). Fig. 2 shows that the number fractions of S-containing dust particles in detected dust particles are $16.7-30.9 \%$, and in most particles $\mathrm{S}$ occupies more than $10 \%$ of weight in total elements of $Z>11$ (Fig. 4b). If $\mathrm{S}$ deposition had occurred 
significantly, there should have been more S-containing dust particles unless $\mathrm{S}$ chose particles to deposit, which is apparently unacceptable. Therefore, $\mathrm{S}$ in these particles is more likely from crustal origins other than sea salt. Similarly, $\mathrm{Na}$ and $\mathrm{Cl}$ in these particles are also most possibly from crustal origins. The reason for this is that there are only a small number of dust particles with their $\mathrm{Na} / \mathrm{Cl}$ close to that of sea salt and their $\mathrm{Na}: \mathrm{S}: \mathrm{Cl}$ are in a small range far apart from that of sea salt (Fig. 4a). If they were formed by a random combination of dust particles and sea salt particles, there should have been more mixture particles along line $l_{1}$ near the position of sea salt. Otherwise, sea salt particles must have chosen dust particles which contained crustal $\mathrm{S}$ to combine, which is also apparently unacceptable.

We quantitatively detected 121, 35 and 67 dust particles for Q-1, Q-2 and Q-3, respectively. Given that all Na- and S-containing particles in which the weight ratio of $\mathrm{S}$ is smaller than $10 \%$ are mixture particles, there are merely 11, 2 and 1 such particles in the analyzed particles of Q-1, Q-2 and Q-3, respectively. This indicates that less than $9 \%$ of Q-1 dust particles, $6 \%$ of Q-2 and $2 \%$ of Q-3 are internally mixed with sea salt, suggesting most dust particles were not interacted by sea salt. Therefore, although $\mathrm{Na}$ and $\mathrm{Cl}$ were detected in a number of dust particles, they are mainly from crustal origins rather than sea salt. The fractions of mixture particles should be much less than $10 \%$ of the dust particles.

\subsection{Sulfate and nitrate formation on dust particles}

The analysis using reagent films revealed that a small number of particles contained sulfate and/or nitrate. The number fractions of reaction spots that had obvious electron-dense cores after reaction among total electrondense particles were estimated from TEM images and the results are listed in Table 2. 3.9-8.4\% of the particles contained sulfate, $5.5-6.9 \%$ contained nitrate, and 1.0 $4.5 \%$ contained both sulfate and nitrate. These figures are also similar to particles observed in Beijing during dust storm periods (Zhang and Iwasaka, 1999). Compared to the results at Qingdao during non-dust periods (Zhang et al., 2000), the fraction of nitrate-containing particles is much smaller during dust storm episodes while the fractions of sulfate- and sulfate\&nitratecontaining particles are not much different. However, in contrast to the fraction of sulfate and nitrate particles in the polluted urban atmosphere in Beijing during nondust periods (Zhang et al., 1995; Zhang, 1996), the fractions are much smaller.

These similarities and differences indicate that the modification of the dust particles by sulfate and nitrate formation from when the particles were emitted until they reached the coastal areas is much weaker than what mineral particles usually experience in polluted urban atmosphere during non-dust periods. Therefore, the enhancement of particulate sulfate and nitrate formation by dust particles may not be significant when the particles are transported in continental air, at least, it should be much weaker than that by mineral particles in polluted urban atmosphere. A possible reason for this is that the air masses containing dust particles are usually very dry (Table 1), which does not favor sulfate and nitrate formation on the particles even assuming that $\mathrm{SO}_{2}$ and $\mathrm{NO}_{x}$ concentrations were the same as in a polluted urban atmosphere.

It should be noted that the above results are not accurate fractions in dust particles. They are for the total detected electron-dense particles. It is known from the elemental analysis that electron-dense particles are mainly dust and sea salt particles. Given that all sea salt particles contained sulfate and none contained nitrate, the number fractions of sulfate- and nitratecontaining dust particles (including dust and sea saltdust mixture particles) were estimated. Note that the given conditions lead to the fraction of sulfate-containing dust particles being a little underestimated and the fraction of nitrate-containing particles being somewhat overestimated. The results are also listed in Table 2 . $3.3-12.2 \%$ (average $7.9 \%$ ) of dust particles containing sulfate and $6.5-10.0 \%$ (average $7.8 \%$ ) containing nitrate

Table 2

Number fractions (\%) of sulfate-, nitrate- and sulfate\&nitrate-containing particles in electron-dense particles detected by reagent film analysis, number fractions of sea salt particles and dust particles detected by element analysis, and the number fractions of sulfate- and nitrate-containing ones in dust particles which are estimated with the assumption that all sea salt particles contain sulfate and none contains nitrate

\begin{tabular}{|c|c|c|c|c|c|c|c|c|c|}
\hline \multirow[t]{2}{*}{ Dust event } & \multicolumn{4}{|c|}{ Reagent film analysis } & \multicolumn{3}{|c|}{ Element analysis } & \multicolumn{2}{|c|}{ In dust particles } \\
\hline & Sulfate & Nitrate & Sulfate\&nitrate & $N_{\mathrm{p}}$ & Sea salt & Dust & $N_{\mathrm{p}}$ & Sulfate & Nitrate \\
\hline Q-1 & 8.4 & 5.5 & 4.5 & 310 & 0.7 & 87.7 & 269 & 12.2 & 10.0 \\
\hline Q-2 & 3.9 & 6.9 & 1.0 & 102 & 0.6 & 92.0 & 175 & 3.3 & 6.9 \\
\hline Q-3 & 8.2 & 6.0 & 0.5 & 184 & 0.5 & 90.3 & 179 & 8.2 & 6.5 \\
\hline Average & & & & & & & & 7.9 & 7.8 \\
\hline
\end{tabular}

$N_{\mathrm{p}}$ are the number of total detected particles in the analysis for each event. 
on their surface. Therefore, $S$ in more than half of $S$ containing dust particles (see Fig. 2) was not in a reactive form even on the surface of the particles, which is consistent with results of elemental analysis. And similar to sulfate, nitrate formation on the particles was not significant either.

\subsection{Size distributions}

To provide more detailed information for accessing the contributions of dust particles to total particulate matters, sizes of dust particles were estimated from their SEM images. The diameter of a particle was defined by the mean of its longest dimension and the orthogonal width. Fig. 5 shows the tentative size distributions of particles analyzed in Fig. 2. The size distribution modes of the particles are around $3 \mu \mathrm{m}$ with ranges of 1.0 $8.0 \mu \mathrm{m}$. Out of this range, dust particles were rarely detected.

From the results of elemental analysis, it is known that the distributions in Fig. 5 are dominated by particles similar to fresh dust particles. No published data at Qingdao are available for comparisons to evaluate the distributions. A recent measurement of particle number-size distributions at Beijing reported by Zhang et al. (2003) indicates that the increases in diameter ranges of rapid concentration during dust storm events are 2.0-5.0 and $0.5-0.7 \mu \mathrm{m}$. Distributions in Fig. 5 are consistent with the former range but we did not find dust particles as defined in the range smaller than $1 \mu \mathrm{m}$. Previous electron microscopic studies of particles collected at Beijing during non-dust storm and dust storm periods revealed that there were a large number of mineral particles in the range smaller than $1 \mu \mathrm{m}$ in the samples. Their shapes and elemental compositions were much different from those of natural

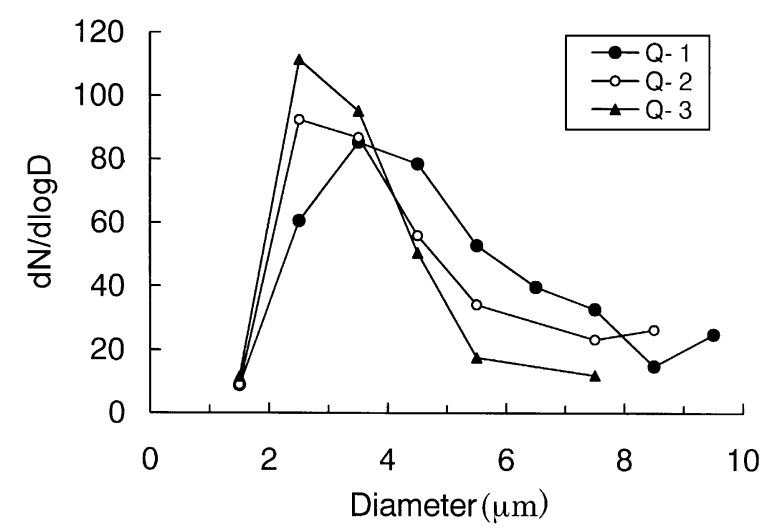

Fig. 5. Tentative number-size distributions of dust particles of the three dust storm events. The distributions were estimated from electron microscope images of the particles detected in Fig. 2 and the total particle numbers in each event were normalized to 100 . dust particles and they were most possibly from anthropogenic sources (Zhang et al., 1995; Zhang and Iwasaka, 1999). In addition, the distribution modes shown in Fig. 5 are approximately consistent with the distributions observed using particle counters or other equipments during dust storm periods in Korea, Japan and even North America (Chun et al., 2001; Husar et al., 2001; Murayama et al., 2001; Tratt et al., 2001; Trochkine et al., 2002).

\section{Summary}

Asian dust particles were collected at a coastal site of Qingdao in northeastern China during three dust storm events in spring 2001. The elemental composition and size of individual dust particles and their mixture state with sulfate, nitrate and sea salt were analyzed. The following results are summarized.

- Even after dust particles completed their travel in the continental atmosphere, more than $90 \%$ of the particles were still similar to fresh dust and they were not disturbed by sulfate, nitrate and/or sea salt.

- Although a number of dust particles contained $\mathrm{Na}, \mathrm{S}$, and $\mathrm{Cl}$, the elements were more likely from crustal origins. $\mathrm{S}$ in more than half of the particles was not on the particle surface or in a reactive form. Nitrate formation on the particles was not significant either.

- Number-size distributions of dust particles estimated from electron microscope images have maxima around $3 \mu \mathrm{m}$ diameter, and the distributions are dominated by particles similar to fresh dust.

Based on these results, we conclude that Asian dust particles may not be interacted considerably by anthropogenic pollutants during their dispersion in the continental atmosphere at northern and northeastern China, and dust particles transported out of the continent have their distribution mode around $3 \mu \mathrm{m}$ diameter. In addition, our results also indicate that the crustal contributions of $\mathrm{Na}, \mathrm{S}$ and $\mathrm{Cl}$ need to be evaluated in the investigation of anthropogenic components in dust particle samples collected in the continental atmosphere.

\section{Acknowledgements}

The authors wish to thank Dr. Y. Ishizaka for his assistance in the TEM analysis. This study was funded by the Japan Society for the Promotion of Science under the Inter-Research Central Cooperative Program and by the Japan Ministry of Education, Science and Technology under the Grant-in-Aid for Scientific Research on Priority Areas (Grant 14048219). The helpful comments 
and suggestions of the two anonymous referees are much appreciated.

\section{References}

Akimoto, H., Narita, H., 1994. Distribution of $\mathrm{SO}_{2}, \mathrm{NO}_{x}$ and $\mathrm{CO}_{2}$ emissions from fuel combustion and industrial activities in Asia with $1^{\circ} \times 1^{\circ}$ resolution. Atmospheric Environment 28, 213-225.

Chun, Y., Kim, J., Choi, J.C., Boo, K.O., Oh, S.N., Lee, M., 2001. Characteristic number size distribution of aerosol during Asian dust period in Korea. Atmospheric Environment 35, 2715-2721.

Dentener, F.J., Carmichael, G.R., Zhang, Y., Lelieveld, J., Crutzen, P.J., 1996. Role of mineral aerosol as a reactive surface in the global troposphere. Journal of Geophysical Research 101, 22869-22889.

Duce, R.A., Unni, C.K., Ray, B.J., Prospero, J.M., Merrill, J.T., 1980. Long-range atmospheric transport of soil dust from Asia to the Tropical North Pacific: temporal variability. Science 209, 1522-1524.

Eriksson, E., 1959. The yearly circulation of chloride and sulfur in nature; meteorological, geochemical, and pedological implications. Part I. Tellus 11, 375-403.

Guttikunda, S.K., Carmichael, G.R., Calori, G., Eck, C., Woo, J.-H., 2003. The contribution of megacities to regional sulfur pollution in Asia. Atmospheric Environment 37, $11-22$.

Husar, R.B., et al., 2001. Asian dust events of April 1998. Journal of Geophysical Research 106, 18317-18330.

Iwasaka, Y., Yamato, M., Imasu, R., Ono, A., 1988. Transport of Asian dust (KOSA) particles; importance of weak KOSA events on the geochemical cycle of soil particles. Tellus 40B, 494-503.

Kanamori, S., Kanamori, N., Nishikawa, M., Higuchi, T., 1991. The chemistry of KOSA (in Japanese). In: WRI of Nagoya University (Eds.), KOSA. Kokin Syoin Press, Nagoya, Japan, pp. 124-156.

Kato, N., Akimoto, H., 1992. Anthropogenic emissions of $\mathrm{SO}_{2}$ and $\mathrm{NO}_{x}$ in Asia: emission inventories. Atmospheric Environment 26A, 2997-3017.

Murayama, T., et al., 2001. Ground-based network observation of Asian dust events of April 1998 in East Asia. Journal of Geophysical Research 106, 18345-18359.

Nishikawa, M., Kanamori, S., Kanamori, N., Mizoguchi, T., 1991. Kosa aerosol as eolian carrier of anthropogenic material. Science of the Total Environment 107, 13-27.

Nishikawa, M., Hao, Q., Morita, M., 2000. Preparation and evaluation of certified reference materials for Asian mineral dust. Global Environmental Research 4, 103-113.

Okada, K., Kai, K., 1995. Features of elemental composition of mineral particles collected in Zhangye, China. Journal of Meteorological Society of Japan 73, 947-957.

Okada, K., Naruse, H., Tanaka, T., Nemoto, O., Iwasaka, Y., Wu, P., Ono, A., Duce, R., Uematsu, M., Merrill, J., 1990. $\mathrm{X}$-ray spectrometry of individual Asian dust-storm particles over the Japanese Islands and the North Pacific Ocean. Atmospheric Environment 24A, 1369-1378.
Posfai, M., Anderson, J.R., Shattuck, T.W., Tindale, N.W., 1996. Constituents of a remote Pacific marine aerosol: a TEM study. Atmospheric Environment 28, 1747-1756.

Qian, G.W., Tanaka, H., Yamato, M., Ishizaka, Y., 1991. Multiple thin film method for simultaneous detection of sulfate and nitrate ions in individual particles and its application to atmospheric aerosols. Journal of Meteorological Society of Japan 69, 629-640.

Sokolik, I.N., Toon, O.B., 1996. Direct radiative forcing by anthropogenic airborne mineral aerosols. Nature 381, 681-683.

Song, H.C., Carmichael, G.R., 2001. A three-dimensional modeling investigation of the evolution processes of dust and sea-salt particles in East Asia. Journal of Geophysical Research 106, 18131-18154.

Tratt, D.M., Frouin, R.J., Westphal, D.L., 2001. April 1998 Asian dust event: a southern California perspective. Journal of Geophysical Research 106, 18317-18379.

Trochkine, D., Iwasaka, Y., Matsuki, A., Zhang, D., Osada, K., 2002. Aircraft borne measurements of morphology, chemical elements, and number-size distributions in the free troposphere in spring over Japan: estimation of particle mass concentrations. Journal of Arid Land Studies 11, 327-335.

Uematsu, M., Duce, R.A., Prospero, J.M., Chen, L., Merrill, J.T., McDonald, R.L., 1983. Transport of mineral aerosol from Asia over the North Pacific Ocean. Journal of Geophysical Research 88, 5343-5352.

Yamato, Y., Tanaka, H., 1994. Aircraft observations of aerosols in the free marine troposphere over the North Pacific Ocean: particle chemistry in relation to air mass origin. Journal of Geophysical Research 99, 5353-5377.

Zhang, D., 1996. Features of individual nitrate-containing particles in the urban atmosphere over Beijing (in Chinese with English abstract). Scientia Atmospherica Sinica 20, 408-413.

Zhang, D., Iwasaka, Y., 1999. Nitrate and sulfate in individual Asian dust-storm particles in Beijing, China in spring of 1995 and 1996. Atmospheric Environment 33, 3213-3223.

Zhang, D., Iwasaka, Y., 2001. Chlorine deposition on dust particles in marine atmosphere. Geophysical Research Letters 28, 3613-3616.

Zhang, D., Tang, X., Qin, Y., Iwasaka, Y., Gai, X., 1995. Tests for individual sulfate-containing particles in urban atmosphere in Beijing. Advances in Atmospheric Sciences 12, 343-350.

Zhang, D., Shi, G., Iwasaka, Y., Hu, M., 2000. Mixture of sulfate and nitrate in coastal atmospheric aerosols: individual particle studies in Qingdao $\left(36^{\circ} 04^{\prime} \mathrm{N}, 120^{\circ} 21^{\prime} \mathrm{E}\right)$, China. Atmospheric Environment 34, 2669-2679.

Zhang, R., Zhang, X., Shi, G., Kanai, Y., Ohta, A., 2003. TSP mass concentration and number concentration of particles in Beijing during dust storms in spring 2002. Proceedings of the Asian Dust Storm and its Impact, 2nd ADEC Workshop, Xian, China, 13-16 January 2003, pp. 12-16.

Zhang, Y., Sunwoo, Y., Kothamarthi, V., Carmichael, G.R., 1994. Photochemical oxidant processes in the presence of dust: an evaluation of the impact of dust on particulate nitrate and ozone formation. Journal of Applied Meteorology 33, 813-824. 